\title{
Aux sources de la grammaire scolaire de Lhomond
}

\author{
Sophie Piron ${ }^{*}$ \\ Université du Québec à Montréal, CP 8888, H3C 3P8 Montréal (Qc), Canada
}

\begin{abstract}
Résumé. L'article porte sur les Élémens de la grammaire françoise de Charles-François Lhomond, publiés en 1780. Il s'intéresse aux sources de ce texte qui a connu un immense succès, sans même avoir innové sur le plan conceptuel. Il s'agit donc d'établir quels modèles ont servi à Lhomond. L'article expose d'abord quelles filiations explicites existent entre Lhomond et la tradition grammaticale. Il se penche ensuite sur le texte des Élémens pour y déceler les filiations non déclarées par Lhomond. L'étude se concentre sur la définition des parties du discours.
\end{abstract}

\begin{abstract}
To the sources of Lhomond's scholar grammar. The article deals with Charles-François Lhomond's Élémens de la grammaire françoise, published in 1780. It studies the sources of this text, which was enormously successful, without having even innovated conceptually. It is therefore a question of establishing which models have served Lhomond. The article first exposes what explicit filiations exist between Lhomond and grammatical tradition. It then looks at the text of the Élémens to detect filiations not declared by Lhomond. The study focuses on the definition of parts of speech.
\end{abstract}

\section{Introduction}

Charles-François Lhomond (1727-1794) a publié en tout sept ouvrages: De Viris illustribus urbis Romae (1779), Élémens de la grammaire latine (1779), Élémens de la grammaire françoise (1780), Doctrine chrétienne, en forme de lectures de piété (1783), Epitome historiae sacrae, ad usum tyronum linguae latinae (1784), Histoire abrégée de l'Église (1787) et Histoire abrégée de la religion avant la venue de J.-C. (1791). On y relève deux grammaires - scolaires - dont les titres se font écho : Élémens de la grammaire latine (EGL) et Élémens de la grammaire françoise (EGF). Toutes deux ont connu un vif succès, déjà du vivant de l'auteur, mais aussi par la suite. Les deux ouvrages ont marqué, à leur manière, l'histoire de la grammaire.

Aucune grammaire française, sans doute, n'a connu un succès aussi durable que celle de Lhomond. (Chervel $1977: 63$ )

La date clé dans l'histoire de la production grammaticale à usage scolaire au XVIII ${ }^{\mathrm{e}}$ siècle n'est pas le Restaut de 1732, qui distingue

\footnotetext{
*Adresse de correspondance : piron.sophie@uqam.ca
} 
les deux orthographes, ni le Wailly de 1754 qui abandonne la déclinaison, mais le petit Lhomond de 1780. (Chervel $2006: 220$ )

En France, ils [les EGL] ont constitué la grammaire latine de référence pendant près d'un siècle [...]. Bien que fondamentalement différents, la méthode de Du Marsais et la grammaire de Lhomond sont sans doute les travaux qui ont le plus marqué l'enseignement du latin au XVIII ${ }^{\mathrm{e}}$ siècle. [...] la première cédera assez rapidement la place à la seconde qui offrira à l'enseignement du latin un fondement d'autant plus incontesté que son caractère traditionnel ne risquait pas de choquer [...]. (Colombat 1999 : 106)

Nous nous intéressons ici aux Élémens de la grammaire françoise (EGF). L'ouvrage a connu sept éditions du vivant de Lhomond, la dernière étant celle de 1790. Les EGF ont ensuite été réédités et adaptés tout au long du XIX ${ }^{\mathrm{e}}$ siècle. Citons par exemple, Lecomte et Ménétrier (1879). L'origine de ce succès ne s'explique pas par la profondeur de l'exposé grammatical. Lhomond ne propose aucun classement novateur, aucune avancée théorique. De ce point de vue, seule l'autonomisation de l'adjectif en partie du discours est à relever, mais Lhomond est loin d'être le premier à repenser le statut du nom adjectif par rapport au nom substantif. Girard (1747), Beauzée (1767), Royon (1777) et Domergue (1778) l'ont précédé sur ce point. Le succès des EGF aura cependant entériné le nouveau classement.

Il faut plutôt chercher ailleurs l'apport de Lhomond. Dans la forme adoptée, d'abord : le style est simple et concis. Dans le contenu proposé, ensuite : la matière est condensée et élaguée. Ce degré zéro du manuel de grammaire (Chervel 2006) a fortement contribué à son succès dans un monde scolaire balbutiant. Les EGF ouvrent la période de la première grammaire scolaire (Chervel 1977), en menant à terme le courant des petites grammaires ou grammaires élémentaires du XVIII' siècle dans lequel ils s'inscrivaient (Piron 2019).

Lhomond étant avant tout un pédagogue, l'essentiel du travail qu'il a produit porte sur la formulation des contenus grammaticaux, leur ordonnancement et leur sélection. Par conséquent, les concepts grammaticaux exposés doivent provenir d'ailleurs. L'objectif de la présente étude est de tenter d'établir les liens de filiation qui doivent exister entre les EGF de Lhomond et la tradition grammaticale du XVIII ${ }^{e}$ siècle, voire antérieure. Il s'agit de montrer chez quels auteurs Lhomond a puisé son inspiration et quelles stratégies d'élagage il a mises en place pour rédiger son ouvrage.

L'article expose d'abord quelles filiations explicites existent entre Lhomond et la tradition grammaticale. Il se penche ensuite sur le texte des EGF pour y déceler les filiations non déclarées par Lhomond. L'étude se concentre sur la définition des parties du discours et prend pour référence la dernière édition parue du vivant de l'auteur, soit celle de 1790 .

\section{Filiations : ce qu'en dit Lhomond lui-même}

Lhomond ne livre pas beaucoup d'informations dans sa préface des EGF pour nous guider à propos des sources qui auraient alimenté la rédaction de sa grammaire. Il semble faire table rase du passé, au moins dans le domaine des ouvrages introductifs comme celui qu'il propose. Il précise que les abrégés existants ne présentent pas les caractéristiques de simplicité nécessaires à un ouvrage pour débutants.

Nous avons de bonnes Grammaires françoises, mais je doute que l'on puisse porter un jugement aussi favorable des Abrégés qui ont été faits pour les Commençans. Les premiers Élémens ne sauroient être trop simplifiés. (Lhomond $1790: 3$ ) 
S'il souligne la qualité d'ouvrages plus ambitieux déjà existants, il n'en nomme aucun dans sa préface. On trouve une seule référence dans le reste des EGF. Il s'agit des Remarques sur la langue françoise (plus spécifiquement, l'opuscule intitulé Essais de grammaire, 1767) de l'abbé d'Olivet, citées en note de bas de page (Lhomond 1790 : 6263) à propos de la règle d'accord du participe passé. D'Olivet sert de caution au choix que pose Lhomond dans deux situations d'accord ${ }^{1}$.

La préface des Élémens de la grammaire latine, parus un an avant les EGF, fournit une autre indication à propos des sources de Lhomond. Celui-ci tient alors à asseoir ses compétences dans le domaine en affirmant être au fait des avancées en grammaire et des critiques adressées à la grammaire traditionnelle.

Je connois les nouveaux plans de Grammaire que l'on propose depuis quelques années, les reproches que l'on fait à la Méthode vulgaire, \& les déclamations peu mesurées que l'on se permet contre ceux qui la suivent. (Lhomond 1781 : ix)

Il est évident que Lhomond se range du côté de «la Méthode vulgaire », des traditionalistes. Il récuse les propositions grammaticales modernes parce qu'elles lui semblent trop abstraites et ne peuvent en ce sens convenir à un ouvrage élémentaire. Lhomond cite alors très exactement Beauzée (1767), en référençant uniquement le titre de la Grammaire générale.

A tout cela, je n'ai qu'un mot à répondre: La Métaphysique ne convient point aux enfans. Quels sont en effet les principes que nous offrent ces nouveaux plans? Les voici fidélement transcrits. Les Noms sont des mots qui expriment déterminément les êtres, en les désignant par l'idée de leur nature: Gramm. Gén. tom. I, pag. 235. (Lhomond $1781:$ ix)

On soulignera par ailleurs que ce modèle récusé dans la préface des Élémens latins n'est pas une grammaire latine. Hormis les rares indications qui viennent d'être relevées, nulle précision explicite de la part de Lhomond sur les sources qu'il aurait exploitées dans ses EGF.

\section{3 Établissement des filiations au sein des EGF}

Pour établir les filiations entre les EGF et les productions grammaticales qui ont précédé celles de Lhomond, nous nous centrerons sur la définition des parties du discours dans les EGF et dans un corpus de 18 grammaires françaises et de 4 éditions du Dictionnaire de l'Académie française (DAF), éditions de 1694, de 1718, de 1740 et de 1762.

\subsection{Orientations fondamentales et choix structurels}

Avant d'entrer dans le cœur du matériau grammatical, il convient de s'interroger sur les orientations fondamentales de l'ouvrage. Elles résident en partie dans la façon dont Lhomond définit la grammaire. Selon lui, «La grammaire est l'art de parler \& d'écrire correctement » (Lhomond 1790:5). Sa définition est en tous points identique à celle de Wailly (1759: 1). Cette conception normative de la grammaire, alliant l'oral et l'écrit, est en accord avec celle que propose le Dictionnaire de l'Académie françoise - dont elle reste étonnamment proche - ou Gaullyer (1722) dans une moindre mesure.

L'art qui enseigne à parler \& à écrire correctement. (DAF 1694, 1718, 1740 et 1762)

La grammaire françoise est l'art d'écrire le françois, de le parler, \& de le prononcer. (Gaullyer $1722: 1$ ) 
L'Académie envisage la grammaire comme la transmission d'une aptitude. Gaullyer, Wailly et Lhomond, pour leur part, ne considèrent que l'aptitude en elle-même et surtout ne l'envisagent que dans sa réalisation physique (l'oral et l'écrit). À cet égard, il est intéressant de comparer leur définition à celles que donnent Panckoucke (1749) et Royon (1777). Si ces derniers adoptent aussi une vision normative, de leur point de vue, la grammaire associe la dimension abstraite des idées à celle, concrète, de leur expression.

C'est l'art d'exprimer ses pensées correctement et avec goût. (Panckoucke $1749: 1$ )

La grammaire est l'art qui donne les principes, pour exprimer correctement ses pensées, tant par le moyen de la parole, que par celui de l'écriture. (Royon $1777: 1$ )

Ainsi, Lhomond, à l'aube de la grammaire scolaire, coupe la grammaire de son objectif de transmission et la réduit à une aptitude qu'il confine, de surcroît, dans l'espace concret de l'oral et de l'écrit normés. La grammaire selon Lhomond n'est pas une théorie du langage, associant le sens à une forme, mais une grammaire de la forme seule.

Sur un autre plan, la notion de partie du discours engage, elle aussi, des choix fondamentaux. À cet égard, la terminologie choisie par Lhomond ne suit pas les dénominations habituelles de parties $d u$ discours ${ }^{2}$ et de parties d'oraison ${ }^{3}$. Lhomond a plutôt porté son choix sur espèces de mots, qui apparaît presque exclusivement dans les titres au sein des EGF.

Chapitre Premier. Premiere espece De mots. Le Nom. (Lhomond $1790: 8)$

Cette terminologie est peu utilisée au XVIII ${ }^{\mathrm{e}}$ siècle. On la trouve, seule ou en synonyme d'autres emplois, chez Buffier (1709), Vallart (1744), Panckoucke (1749), D’Olivet (1767) et Beauzée (1767).

Lhomond retient ensuite l'adjectif dans la liste des espèces de mots, comme l'ont fait avant lui Girard (1747), Beauzée (1767), Royon (1777) et Domergue (1778). Cette autonomisation impose de faire un choix terminologique pour le nom substantif dont est désormais disjoint le nom adjectif. Si Girard et Domergue ont retenu l'hyponyme substantif, Lhomond choisit de suivre Beauzée (que suit aussi Royon 1777). Il adopte donc l'hyperonyme nom, désigné comme vulgaire par Girard (1747) et vraisemblablement en usage dans l'enseignement primaire élémentaire.

La grammaire nomme cette sorte de mots Noms \& SUBSTANTIFS. Le premier de ces termes plus vulgaire est précisément pris du but de leur institution [...]. (Girard 1747, t. $1: 217$ )

[C]'est donc à juste titre qu'on a nommé les mots de cette espece Noms \& SubSTANTIFS, termes dont la valeur est la même à quelque accessoire près, qu'il n'est pas question d'expliquer ici. Quoique le dernier puisse paroitre étranger à des oreilles qui n'ont pas fréquenté le collége ; je m'en servirai néanmoins ; parceque c'est le terme consacré dans les Dictionnaires et chez les Grammairiens qui m'ont précédé [...]. (Girard 1747, t. $1: 45$ )

Lhomond opte également pour un ordre de présentation des espèces de mots qui positionne la description de l'article après celle du nom, suivant en cela ce que recommande D'Olivet.

Puisque l'article sert uniquement à modifier les noms, il m'a paru d'une indispensable nécessité, que l'on se fût mis au fait de ce qui concerne les noms avant que d'étudier ce qui regarde l'article. (D’Olivet $1767: 139$ ) 
Cet ordre n'est pas systématiquement suivi à l'époque, mais il est loin d'être exceptionnel. L'ont ainsi adopté des petites grammaires comme celles de Restaut (1732), Wailly (1759), Viard (1763), Bertera (1773), et des grammaires des dames comme celles de Panckoucke (1749) et De Prunay (1777). Toujours concernant l'ordre, Lhomond expose la préposition avant l'adverbe dans ses EGF, à l'inverse de ce qu'il propose dans ses Élémens latins. L'ordre préposition/adverbe est adopté par Wailly (1759), Beauzée (1767), De Prunay (1777), Royon (1777). Tant dans le cas de l'article que dans celui de la préposition, Lhomond suit un choix qu'avait déjà posé Port-Royal (1660).

La terminologie espèce de mots et l'ordre de description des parties du discours positionnent Lhomond dans une veine grammaticale plus accessible, mais ne livrent aucun indicateur ferme de filiation.

\subsection{La définition des espèces de mots : le nom}

Lhomond définit le nom en reprenant presque mot à mot la définition de Wailly, mais il la rend encore plus concrète par l'utilisation du déterminant indéfini singulier dans sa valeur d'exemple (une personne, une chose), plutôt que d'employer le déterminant pluriel généralisant (les personnes, les choses).

Le Nom est un mot qui sert à nommer une personne ou une chose, comme Pierre, Paul, Livre, Chapeau. (Lhomond $1790: 8$ )

Les substantifs servent à nommer les personnes ou les choses. (Wailly $1759: 2)$

L'optique choisie rejoint la définition en langue générale que propose le DAF pour le nom, et non la définition en grammaire.

Le terme dont on a accoutumé de se servir pour désigner chaque personne, chaque chose. [...] En Grammaire, se dit d'Un mot susceptible de nombre \& de genre. (DAF 1762, nom)

Terme de Grammaire. Il se dit, De tout nom qui signifie quelque substance, quelque être, quelque chose que ce soit; \& qui peut s'employer dans le discours sans le secours d'aucun autre nom. (DAF 1762, substantif)

Dans la même optique, des grammairiens mondains (D’Olivet 1767, De Prunay 1777) considèrent que le nom permet de désigner. Le courant des petites grammaires choisit plutôt la capacité du nom à nommer (Restaut 1732, Wailly 1759, Viard 1763, Royon 1777, Chompré et al. 1778). Le choix terminologique du nom s'en trouve motivé et renforcé. Seuls Girard (1747) et Wailly (1759) continuent d'utiliser le terme substantif, tout en le définissant par sa capacité à nommer.

\subsection{La définition des espèces de mots : l'article}

La tradition définit très souvent l'article comme une particule (DAF 1694 à 1762 ; RégnierDesmarais 1707 ; Buffier 1709 ; Gaullyer 1722). Lhomond s'inscrit dans une approche plus intuitive et suit les définitions proposées par des abrégés (Restaut 1732, Viard 1763, Chompré et al. 1778, également la grammaire des dames de De Prunay 1777) : l'article est un petit mot. La définition des EGF est calquée sur celle de Restaut (lui-même s'inspirant de Gaullyer 1722), hormis la référence aux cas.

L'Article est un petit mot que l'on met devant les noms communs, \& qui en fait connoître le genre \& le nombre. (Lhomond $1790: 8$ ) 
Ce sont de petits mots qui se mettent avant les noms, \& qui en font ordinairement connoître le genre, le nombre, \& le cas. (Restaut 1732 : 14)

[L]es articles ne sont autre chose que des particules déclinables qui precedent toujours le nom auquel elles se joignent, \& qui servent à en faire connoître le genre \& le nombre. (Gaullyer $1722: 4$ )

Lhomond a ajouté dans sa définition la restriction selon laquelle l'article ne concerne que les noms communs. Cette précision ne se trouve que dans le DAF de 1762, pas avant.

En Grammaire, est Une particule qui se met devant le nom, pour en marquer le genre, le nombre, \& le cas. (DAF 1740, article)

En Grammaire, est une particule qui précéde ordinairement les noms appellatifs. (DAF 1762, article)

\subsection{La définition des espèces de mots : le pronom}

Lhomond définit le pronom très simplement en ne faisant appel qu'à la question du remplacement du nom. Il laisse de côté l'économie de répétition que soulignent des grammairiens comme Vallart (1744) ou Wailly (1759). Sa source est, ici encore, Restaut, qui utilise l'expression tenir la place (reprise par Vallart 1744) alors que les autres ouvrages utilisent mettre à la place (DAF 1694 à 1762, Wailly 1759, D’Olivet 1767).

Le pronom est un mot qui tient la place du nom. (Lhomond $1790: 18$ )

C'est un mot qui tient ordinairement la place du nom. (Restaut 1732 :

32)

\subsection{La définition des espèces de mots : le verbe}

La définition du verbe que proposent les EGF s'écarte des modèles suivis pour les autres parties du discours. Les modèles habituels que sont Restaut et Wailly ne peuvent, cette fois, convenir à Lhomond, puisque leurs définitions sont colorées par la grammaire générale, où le verbe est une affirmation ou un jugement. Or, rappelons-le, la définition que Lhomond a donnée de la grammaire, la place uniquement dans un espace de la forme, en dehors du sens. Lhomond n'a pas choisi non plus l'orientation prise par les différentes éditions du DAF, qui insistent sur la morphologie verbale (marques de temps, de mode et de personne). Il a plutôt opté pour une définition ancrée dans une vision notionnelle des parties du discours, plaçant une fois de plus la grammaire dans le concret, dans la compréhension transparente du métalangage. Régnier-Desmarais (1707), Buffier (1709), Vallart (1744) et Girard (1747) avaient emprunté cette voie avant lui. Lhomond s'est ici très nettement inspiré de Buffier, en expurgeant la définition de ses renvois à la grammaire philosophique (ce qu'on affirme du sujet).

Le verbe est un mot dont on se sert pour exprimer que l'on est ou que l'on fait quelque chose. (Lhomond $1790: 24$ )

Le verbe est un mot qui sert ou peut servir à exprimer ce qu'on affirme du sujet. Ce qu'on peut en affirmer se réduit 1) à ce qu'il est 2) à ce qu'il fait. (Buffier $1709: 64$ )

Le verbe est un mot qui sert à exprimer ce qu'une chose est, ce qu'elle fait ou ce qu'elle reçoit ou souffre. (Vallart $1744: 216$ )

\subsection{La définition des espèces de mots : l'adjectif}


Lhomond a davantage modifié ses modèles pour définir l'adjectif, et ce, de manière à rendre l'expression la plus concrète possible. Ici encore, il opte pour le déterminant indéfini une, plus concret.

L'Adjectif est un mot que l'on ajoute au nom pour marquer la qualité d'une personne ou d'une chose. (Lhomond $1790: 12$ )

Si l'on sent l'influence générale de Girard (1747), les véritables sources de Lhomond se situent ailleurs. Il a composé une définition qui repose sur D'Olivet (1767) pour l'aspect syntaxique - il s'ajoute au nom pour marquer quelque chose - et sur Wailly (1759) pour l'aspect notionnel - la qualité des personnes \& des choses.

Ceux [les mots] qu'on emploie à marquer les qualités se nomment ADJECTIFS ; parcequ'ils sont ajoutés \& unis aux substantifs pour qualifier les choses que ceux-ci dénomment. (Girard 1747, tome 1 : 48)

On appelle Adjectif, le nom qui s'ajoute au substantif pour le qualifier, c'est-à-dire, pour marquer ce qu'il a de propre ou d'accidentel. (D'Olivet 1767 : 148)

L'Adjectif exprime la qualité des personnes \& des choses. (Wailly $1759: 8)$

\subsection{La définition des espèces de mots : le participe}

Le participe est présenté dans les EGF de manière très différente par rapport aux Élémens latins, où il est un adjectif issu d'un verbe qui s'accorde en genre, en nombre et en cas. En français, il est défini à partir de sa double nature de verbe et d'adjectif. Alors que beaucoup de grammaires motivent la terminologie en disant que le participe participe du nom (adjectif) et du verbe, Lhomond a opté pour l'expression tenir de, que l'on ne trouve que dans les définitions proposées par Gaullyer (1722), DAF (1762) et Royon (1777). Lhomond a dû, comme pour le nom et l'article, s'inspirer de l'édition de 1762 du DAF. C'est toutefois chez Vallart (1744) que se trouvent les éléments de définition retravaillés soit la signification et le régime du verbe, ainsi que la qualification d'un nom - mais surtout émondés par Lhomond.

Le Participe est un mot qui tient du verbe \& de l'adjectif, comme aimant, aimé : il tient du verbe en ce qu'il en a la signification \& le régime : aimant Dieu, aimé de Dieu; il tient aussi de l'adjectif, en ce qu'il qualifie une personne ou une chose, c'est-à-dire qu'il en marque la qualité. (Lhomond $1790: 60$ )

Partie d'oraison qui est un membre de l'infinitif. On l'appelle Participe, parce que c'est un mot qui tient du nom et du verbe tout à la fois. Il tient du nom en ce qu'il est adjectif \& déclinable. Il tient du verbe deux propriétés, celle de marquer le temps, \& celle de régir. (DAF 1762, Participe)

Les participes sont des adjectifs formez des verbes dont ils prennent la signification \& le régime, comme, Aimant la vertu, estant estimé des honnestes gens. Les participes sont ainsi appelez parce qu'ils participent de la nature du nom \& de la nature du verbe. Ils participent de la nature du nom en ce qu'ils servent toujours à qualifier un nom substantif, \& qu'en certaines occasions, ils ont deux terminaisons, l'une masculine et l'autre féminine [...]. Ils participent de la nature du verbe en ce que non seulement ils en ont la signification \& le régime, mais encore les deux voies actives \& passives, avec divers temps. (Vallart $1744: 160-161$ ) 
Les apports de Lhomond, outre le resserrement de la définition sur les aspects essentiels, consistent d'abord à avoir inversé l'ordre adjectif-verbe dans la double nature du participe, insistant ainsi sur l'origine verbale du mot. Lhomond semble être le seul à avoir adopté cet ordre dans la définition du participe. L'autre apport de Lhomond est d'avoir concrétisé la description de la qualification en adoptant un point de vue notionnel et référentiel. En effet, chez Vallart, le participe qualifie un nom alors que chez Lhomond, il qualifie une personne ou une chose. Lhomond fait ici écho à sa propre définition de l'adjectif, qui «marque la qualité d'une personne ou d'une chose » (Lhomond $1790: 3$ ). On voit le souci pédagogique de cohérence au sein des EGF.

\subsection{La définition des espèces de mots : l'adverbe}

La définition de l'adverbe est atypique dans le processus rédactionnel de Lhomond. D'une part, Lhomond ne puise pas auprès de ses sources habituelles, d'autre part il confère à cette partie du discours un caractère plus abstrait. Cette fois, c'est dans la Nouvelle méthode latine de Port-Royal que l'on trouve la filiation pour le cœur de la définition (l'adverbe détermine la signification). Le reste est, une fois encore, inspiré de la $4^{\mathrm{e}}$ édition du DAF (l'adverbe se joint aux verbes et aux adjectifs). Il est à signaler que Lhomond restreint la portée de l'adverbe aux seuls verbes et adjectifs, ce que proposent uniquement le DAF et Vallart (1744).

L'Adverbe est un mot qui se joint ordinairement au verbe ou à l'adjectif, pour en déterminer la signification; quand on dit: cet enfant parle distinctement, par ce mot distinctement l'on fait entendre qu'il parle d'une manière, plutôt que d'une autre. (Lhomond 1790 : 67)

L'adverbe est un mot indéclinable qui, estant joint aux autres, détermine ou spécifie leur signification. (Lancelot $1644: 59$ )

Partie indéclinable de l'oraison, qui se joint avec les verbes \& avec les adjectifs, pour en exprimer les manières ou les circonstances. (DAF 1762)

Lhomond ne s'inscrit donc pas dans le courant qui fait de l'adverbe l'expression d'une circonstance ou d'une modification. Il récuse sur ce point des modèles qu'il suit ailleurs, Restaut 1732, Vallart 1744 et Wailly 1759 . On vient d'ailleurs de le voir, il n'a pas repris cette partie de la définition du DAF. Une fois de plus, Lhomond tente d'ancrer son texte dans le concret.

C'est un mot indéclinable qui se joint ordinairement au verbe pour en exprimer quelque circonstance. (Restaut $1732: 122$ )

Or les qualitez des choses exprimées par les adjectifs, \& les actions signifiées par les verbes, sont susceptibles de certaines circonstances qui s'expriment par des mots particuliers, que l'on nomme adverbes. Ainsi l'adverbe est un mot dont l'usage est de modifier un nom adjectif ou un verbe. (Vallart $1744: 370$ )

L'ADVERBE exprime quelque circonstance du Nom, du Verbe ou même d'un autre Adverbe auquel il a raport. (Wailly $1759: 72$ )

\subsection{La définition des espèces de mots : la préposition}

Lhomond semble avoir innové dans son approche de la préposition. Il s'éloigne de toutes les définitions en place, y compris de ce qu'il a adopté dans les Élémens latins. Dès lors, il ne présente pas la préposition comme un terme en régissant un autre. Il n'établit pas non 
plus l'équivalence entre un adverbe et une séquence $<$ préposition + mot régi $>$. Il prépare un parallélisme qu'il est le seul à tracer au cœur même des définitions. Il rapproche la préposition de la conjonction, toutes deux étant des mots de jonction.

La préposition est un mot qui sert à joindre le nom ou pronom au mot qui la précède. (Lhomond $1790: 64$ )

La conjonction sert à joindre une phrase à une autre phrase [...]. (Lhomond $1790: 68-69$ )

Il est vrai que l'on décèle cette notion de jonction dans la préposition essentiellement chez trois grammairiens : chez Régnier-Desmarais (1707), superficiellement lors d'une réflexion introductive, et donc pas dans la définition même de la préposition ; chez Girard (1747) dans la conception du rapport déterminatif; et surtout chez Beauzée (1767). Lhomond y a fort vraisemblablement puisé son inspiration. Les constructions qu'il présente (le fruit de l'arbre, utile à l'homme, j'ai reçu de mon père) détaillent les mêmes séquences que chez Beauzée (nom : la main de Dieu; adjectif : utile à la nation; verbe : penser avec justesse).

Les deux parties d'oraison [préposition et conjonction] dont il nous reste à parler sont en quelque sorte les nerfs et les ligaments de tout le discours [...]. Tous les autres Termes sont comme les materiaux préparez pour la construction d'un bastiment; Les Prépositions sont comme le ciment \& la chaux [...]. (Régnier-Desmarais $1707: 556$ )

Cette indication de raport supose nécessairement d'autres mots à leur suite \& sous leur régime, pour être le complément du raport \& en former le sens en entier. (Girard 1747, tome $2: 181$ )

Les prépositions sont des mots qui désignent des rapports généraux, avec abstraction de tout terme antécédent \& conséquent. (Beauzée 1767 , tome $1: 515$ )

Au fil de ses exemples, Lhomond signale que la préposition marque le rapport qu'il y a entre deux mots.

Le fruit de l'arbre; de marque le rapport qu'il y a entre fruit \& arbre [...]. (Lhomond $1790: 64$ )

On y perçoit la tradition port-royaliste, à laquelle les EGF empruntent également la division de la syntaxe en syntaxe de convenance et de régime. Or l'innovation de Lhomond dans la définition de la préposition lui permet de solidifier la syntaxe de régime, qui constitue une sorte de charpente au sein des EGF. Elle prend place aux côtés de la syntaxe de convenance et, ensemble, elles structurent en partie la description des espèces de mots. La syntaxe de régime intervient notamment dans la description des adjectifs, des verbes et des conjonctions. Syntaxe de régime et prépositions vont donc de pair chez Lhomond ${ }^{4}$, et cette association est renforcée par la définition de la préposition.

Régime des adjectifs. Regle. Pour joindre un nom à un adjectif précédent, on met de ou $\grave{a}$ entre cet adjectif \& le nom : alors on appelle ce nom le régime de l'adjectif. [...] La syntaxe de régime, par laquelle un mot régit $d e$ ou à devant un autre mot. (Lhomond 1790 : 15)

\subsection{La définition des espèces de mots : la conjonction}

Ici aussi, Lhomond contrevient à la définition de ses Élémens latins et à presque toutes les définitions en usage. La majorité des grammairiens font de la conjonction un mot qui lie. C'est d'ailleurs l'option que Lhomond avait choisie dans les Élémens latins: «La 
conjonction est un mot indéclinable qui sert à lier les parties du discours. " (Lhomond 1781 : 96). Cette fois, il ne conserve pas le strict parallélisme entre ses EGL et ses EGF. D'abord, il choisit le verbe joindre, comme Gaullyer (1722), Wailly (1759) et les différentes éditions du DAF. Sur ce point, la terminologie est désormais motivée en exploitant la même racine conjonction/joindre. Ensuite, et c'est là la rupture la plus importante, Lhomond fait de la conjonction un mot qui joint des phrases, répartissant ainsi les types de jonctions entre la préposition et la conjonction. Or c'est chez Beauzée (1767) et surtout chez Girard (1747) que se trouve cette conception de la conjonction.

La conjonction sert à joindre une phrase à une autre phrase $[\ldots]$. (Lhomond $1790: 68-69$ )

$[\mathrm{U}]$ ne partie d'oraison qui joint les membres du discours. (DAF 1694, $1718,1740,1762)$

De sorte que ces mots, qu'on a nommés CONJONCTIONS, sont proprement la partie systématique du Discours ; puisque c'est par leur moyen qu'on assemble des frases, qu'on lie les sens, \& que l'on compose un tout de plusieurs portions, qui, sans cette neuvieme espece, ne paroitroient que comme des énumérations ou des listes de frases, \& non comme un ouvrage suivi \& affermi par les liens de l'analogie. (Girard 1747, tome $2: 257-258$ )

Plusieurs conjonctions semblent, au premier aspect, ne servir qu'à lier un mot avec un autre: mais si l'on y prend garde de près, on verra qu'en effet elles servent à lier les propositions partielles qui constituent l'ensemble d'un même discours." (Beauzée 1767, tome 1 : 564)

\subsection{La définition des espèces de mots : l'interjection}

Lhomond a ajusté la définition de l'interjection dans ses EGF par rapport à ses Élémens latins (EGL), dans lesquels on sent l'influence de Port-Royal (1660) et de Wailly (1759).

L'interjection est un mot indéclinable qui sert à marquer les différents mouvements de l'ame. (Lhomond 1781, EGL : 97)

Les interjections sont des mots qui ne signifient aussi rien hors de nous : mais ce sont seulement des voix plus naturelles qu'artificielles, qui marquent les mouvements de notre ame, comme ha, o, heu, helas, \&c. (Arnauld et Lancelot $1660: 140$ )

Les particules servent à marquer une affection ou un mouvement de l'ame, soit de douleur \& de tristesse [...]. (Wailly $1759: 74$ )

Dans les EGF, l'interjection est toujours une partie du discours en lien avec l'âme, mais la définition est désormais plus concrète grâce au verbe exprimer (au lieu de marquer) et à l'expression les sentiments de l'âme (plutôt que les mouvements de l'âme). Elle est aussi désormais plus concrète grâce aux deux exemples de mouvements de l'âme que fournit désormais Lhomond.

L'interjection est un mot dont on se sert pour exprimer un sentiment de l'ame, comme la joie, la douleur, \&c. (Lhomond 1790, EGF : 70)

Les grammaires qui usent du verbe exprimer font partie des références que Lhomond a utilisées ailleurs : Restaut (1732) et Vallart (1744). Mais c'est surtout l'expression en usage dans le DAF, depuis la première édition. Les deux exemples de sentiments proviennent de chez Restaut (1732), ce sont les deux premiers de sa liste, dans cet ordre. 
L'une des parties d'oraison dont on se sert pour exprimer les passions, comme la douleur, la colère, la joie, l'admiration, \&c. Ha ! Helas ! (DAF 1718, 1740, 1762)

Ce sont des mots indéclinables dont on se sert pour exprimer quelques mouvements de l'ame, comme la joie, la douleur, la crainte, l'aversion, l'encouragement, \&c." (Restaut $1732: 117)$

La suite de la définition de Lhomond doit probablement beaucoup à Beauzée (1767), qui est le seul à utiliser le terme de sentiment.

Les interjections [sont] des signes naturels \& non d'institution [...]. Il $\mathrm{y}$ a donc en effet des parties d'Oraison de deux espèces : les premières sont les signes naturels des sentiments [...] celles-là constituent le langage du cœur, elles sont affectives [...]. (Beauzée 1767, tome 1 : 611)

\section{Conclusion}

L'étude des filiations qui vient d'être menée a montré que Lhomond a puisé à des sources diverses pour rédiger les définitions des parties du discours dans ses EGF : Wailly (définition de la grammaire, du nom, de l'adjectif), Beauzée (terme nom plutôt que celui de substantif; définition de la préposition et de l'interjection), 4 édition du DAF (définition du nom, du participe, de l'adverbe), Restaut (définition de l'article, du pronom), Buffier (définition du verbe), Vallart (définition du participe), D’Olivet (définition de l'adjectif), Lancelot (définition de l'adverbe), Girard (définition de la conjonction). La filiation est nette pour le nom, l'article, le pronom et le verbe. Souvent, Lhomond entremêle ses sources pour aboutir à une définition concise et concrète. Le travail stylistique et pédagogique est davantage marqué pour l'adjectif, le participe, l'adverbe, et encore plus dans le cas de la conjonction et de l'interjection. De toutes les parties du discours, la préposition est celle qui fait l'objet d'un important travail de réécriture des sources. C'est une pièce maîtresse dans les EGF puisqu'elle est au cœur de la syntaxe de régime telle que la définit Lhomond. Les sources utilisées montrent que plans nouveaux de grammaire et méthode vulgaire ont été entremêlés, mais retravaillés, pour produire un condensé grammatical accessible.

\section{Références bibliographiques}

Académie française. (1694) Dictionnaire de l'Académie française. Paris : Coignard.

Académie française. (1718) Dictionnaire de l'Académie française. Paris : Coignard.

Académie française. (1740) Dictionnaire de l'Académie française. Paris : Coignard.

Académie française. (1762) Dictionnaire de l'Académie française. Paris : Brunet.

Arnauld, A., Lancelot, C. (1660). Grammaire generale et raisonnée. Paris : Pierre le Petit.

Beauzée, N. (1767). Grammaire générale, ou Exposition raisonnée des éléments nécessaires du langage, Pour servir de fondement à l'étude de toutes les langues. Paris : Barbou.

Bertera, B. A. (1773). Nouvelle grammaire, contenant en abrégé tous les principes de la langue française. Paris : Vve Vallat-la-Chapelle.

Buffier, C. (1709). Grammaire françoise sur un plan nouveau, Paris, Nicolas Le Clerc.

Chervel, A. (1977). Et il fallut apprendre à écrire à tous les petits Français. Histoire de la grammaire scolaire. Paris : Payot.

Chervel, A. (2006). Histoire de l'enseignement du français $d u X V I I^{e}$ au XX $X^{e}$ siècle. Paris : Retz, Usuels. 
Chompré, É.-M., Monchablon E.-J., Batteux Ch. (1778). Petite grammaire françoise, à l'usage des élèves de l'École royale militaire. Paris : Nyon aîné.

Colombat, B. (1999). La grammaire latine en France à la Renaissance et à l'Âge classique. Grenoble : Ellug.

D’Olivet, P. J. (1767). Remarques sur la langue françoise. Paris : Barbou.

Domergue, U. (1778). Grammaire françoise simplifiée. Lyon : l'auteur.

Gaullyer, D. (1722). Abrégé de la grammaire françoise. Paris : Jean Baptise Brocas.

Girard, G. (1747). Les vrais principes de la Langue Françoise. Paris : Le Breton.

Lancelot, Claude (1644). Nouvelle Méthode pour apprendre facilement, \& en peu de temps la langue latine. Paris : Antoine Vitré.

Lecomte, E., Ménetrier abbé (1879). Grammaire française de Lhomond complétée et mis dans un ordre meilleur, $23^{\mathrm{e}}$ édition revue et corrigée. Paris : Lecoffre fils.

Lhomond, Ch.-F. (1781 [1779]). Élémens de la grammaire latine. Paris : Colas.

Lhomond, Ch.-F. (1790 [1780]). Élémens de la grammaire françoise. Paris : Colas.

Panckoucke, A.-J. (1749). Les études convenables aux demoiselles. Lille : Panckoucke.

Piron, S. (2019). Lhomond et ses prédécesseurs, in Fournier, J.-M., Raby, V., Lahaussois, A. (éd.), Grammaticalia. Hommage à Bernard Colombat, Lyon : ENS Éditions, 155-163.

Prunay, Mr de. (1777). Grammaire des Dames, Paris, Lottin l'aîné.

Regnier Desmarais, abbé (1705). Traité de la grammaire françoise. Paris : Jean Baptiste Coignard.

Restaut, P. (1732). Abrégé des principes de la grammaire françoise. Paris : Desaint.

Royon. (1777). Traité élémentaire de grammaire et d'orthographe française. Paris : D.-C. Couturier.

Vallart, J. (1744). Grammaire françoise. Paris : Desaint et Saillant.

Viard, N.-A. (1763). Les vrais principes de la lecture, de l'orthographe et de la prononciation françoise. Paris : Panckoucke.

De Wailly, N. F. (1759). Abrégé de la grammaire françoise. Paris : De Bure et Barbou.

${ }^{1}$ La leçon que vous ont donnée vos maîtres (et non donné ; accord du participe passé même en présence d'un sujet inversé) et Adam et Ève que Dieu avoit créés innocens (et non créé; accord du participe passé même en présence d'un attribut de l'objet après le participe).

${ }^{2}$ Excepté à un endroit: «Il y a en françois dix sortes de mots qu'on appelle les parties $d u$ discours $\gg$ (Lhomond $1790: 7$ ).

${ }^{3}$ C'est le choix du DAF 1694, 1718, 1740 et 1762.

${ }^{4}$ C'était le cas aussi dans Port-Royal (1660), mais les cas étaient également considérés : «La Syntaxe de regime au contraire, est tout arbitraire, \& par cette raison se trouve très-differente dans toutes les Langues. Car les unes font les regimes par les cas; les autres au lieu de cas ne se servent que de petites particules qui en tiennent lieu, \& qui ne marquent même que peu de ces cas, comme en François \& en Espagnol on a que $D e, \& A$, qui marquent le genitif \& le datif. [...] On peut voir sur ce sujet ce que nous avons dit cy-dessus, des prepositions \& des cas. (Arnauld \& Lancelot 1660 : 141142). 